\title{
Expression of hepatocyte growth factor in breast cancer and its effect on prognosis and sensitivity to chemotherapy
}

\author{
HUA YANG, CHONGJIAN ZHANG and SHUDE CUI \\ Department of Breast Surgery, Henan Tumor Hospital, Zhengzhou, Henan 450003, P.R. China
}

Received February 9, 2014; Accepted September 29, 2014

DOI: $10.3892 / \mathrm{mmr} .2014 .2808$

\begin{abstract}
The aim of the present study was to examine the expression of hepatocyte growth factor (HGF) in breast cancer and its effect on prognosis and sensitivity to chemotherapy. Immunohistochemistry was conducted to determine the expression of HGF in 125 breast cancer patients. The correlation between the expression level of HGF and the effect of preoperative chemotherapy or 5-year survival rate was then investigated. The human breast cancer cell line, MCF-7, was transfected with a HGF-small interfering (si) RNA interference sequence. Reverse transcription quantitative polymerase chain reaction and western blot analysis were used to confirm the interference efficiency of HGF-siRNA. An MTT assay was used to detect the proliferative activity of MCF-7 cells following silencing of HGF and during co-culture with epirubicin (EPI) at different concentrations. HGF was highly expressed in breast cancer patients and was not associated with patient age, location, size or hormone receptor status of the tumor $(\mathrm{P}>0.05)$, however, HGF expression was associated with tumor-node-metastasis (TNM) clinical stage, histological grade, lymph node metastasis and prognosis $(\mathrm{P}<0.05)$. The efficiency of chemotherapy in HGF negative patients $(90 \%)$ was significantly higher $(\mathrm{P}<0.05)$ compared with HGF positive patients (68.75\%). Following successful downregulation of HGF by HGF-siRNA, the tolerance to EPI decreased in MCF-7 cells. In conclusion, HGF was highly expressed in breast cancer cells and was closely associated with lymph node metastasis, prognosis and sensitivity to chemotherapy. Therefore, HGF may be a potential indicator of the prognosis and effectiveness of chemotherapy for breast cancer.
\end{abstract}

Correspondence to: Dr Shude Cui, Department of Breast Surgery, Henan Tumor Hospital, 127 Dongming Road, Zhengzhou, Henan 450003, P.R. China

E-mail: shudecui@163.com

Key words: breast cancer, hepatocyte growth factor, neoadjuvant chemotherapy, prognosis

\section{Introduction}

The burden of breast cancer continues to increase according to global cancer statistics (1). Breast cancer is the most frequently diagnosed cancer and the leading cause of cancer-associated mortality among females. Systemic chemotherapy is an important type of complementary treatment for breast cancer. As the effectiveness of chemotherapy significantly decreases, even leading to resistance, it is crucial to identify definite predictors of sensitivity to chemotherapy, including the widely accepted factors programmed cell death protein 5, human epidermal growth factor receptor 2 and topoisomerase II $\alpha$ (2). Hepatocyte growth factor (HGF) belongs to the family of plasminogen-related growth factors (PRGFs) and is also called PRGF-1. As evidenced by previous studies, HGF is the ligand of the c-Met receptor and is a multifunctional cytokine, which is involved in tumor cell-cell interactions, matrix adhesion, migration, invasion and angiogenesis (3-6).

Breast cancer cells can produce HGF that acts in a paracrine as well as in an autocrine manner (7). HGF is able to induce CXC chemokine receptor 4 expression and contributes to tumor cell invasiveness in breast cancer and the c-Met-HGF axis can enhance the metastatic behavior of breast cancer cells (8). Serum levels of HGF in breast cancer patients were significantly increased when compared with controls (6). It was reported that patients with more advanced tumor-node-metastasis (TNM) staging have higher serum soluble HGF levels (9). Furthermore, HGF/Met signaling was reported to be involved in breast cancer progression (10). However, to the best of our knowledge, few studies have demonstrated that HGF is expressed in breast cancer and the possibility that HGF may be a potential predictor of the effectiveness of chemotherapy remains to be elucidated $(4,11)$. The present study was designed to confirm the expression profile of HGF in breast cancer tissues from 125 patients and in breast cancer cells, and to elucidate the possible association between the expression level of HGF, the effectiveness of chemotherapy and the prognosis of patients.

\section{Materials and methods}

Clinical data. Samples were collected from 125 patients diagnosed with breast cancer through breast biopsy prior to chemotherapy at the Henan Tumor Hospital (Zhengzhou, China) between June 2008 and June 2010. The pathological findings of the patients were analyzed following surgery. Of 
the 125 patients, 62 received 1 week presurgical chemotherapy among whom 41 patients adopted the cyclophosphamide (CTX) epirubicin (EPI) and 5-fluorouracil (5-FU) regimen, including intravenous injection of CTX at $800 \mathrm{mg} / \mathrm{m}^{2}$ on day 1 and 8 , intravenous drip of EPI at $60 \mathrm{mg} / \mathrm{m}^{2}$ on day 2 and 3 and an intravenous drip of $5-\mathrm{FU}$ at $500 \mathrm{mg} / \mathrm{m}^{2}$ during day 4 and 6 . In total, 21 adopted the cyclophosphamide (CTX) tetrahydrofuran (THF) and 5-FU regimen, including intravenous injection of CTX at $800 \mathrm{mg} / \mathrm{m}^{2}$ on day 1 and 8 , intravenous drip of THF at $30 \mathrm{mg} / \mathrm{m}^{2}$ on day 2 and 3 and an intravenous drip of 5-FU at $500 \mathrm{mg} / \mathrm{m}^{2}$ during day 4 and 6 . All the 125 patients were female and the average age was $44.5 \pm 6.3$ years. The histopathological types were as follows: 92 patients had invasive ductal carcinoma, 18 invasive lobular carcinoma, 3 tubular carcinoma, 2 papillary carcinoma, 4 mucous carcinoma and 6 typical medullary carcinoma. In addition, 52 patients had postoperative lymph node metastasis and 76 were estrogen-receptor (ER) (+) and 71 were progesterone receptor (PR) $(+)$. Their histological grades were as follows: 94 were grade I and II and 31 were grade III. Their TNM clinical stages were as follows: 87 were stage I and II and 38 were stage III. Follow-up data of 112 patients were completed. The present study was conducted in accordance with the declaration of Helsinki and with approval from the Ethics Committee of Henan Tumor Hospital. Written informed consent was obtained from all participants.

Cell culture and transfection. The human breast cancer cell line, MCF-7, was purchased from the Shanghai Cell Bank of the Chinese Academy of Sciences (Shanghai, China). The MCF-7 cells were cultured in Dulbecco's modified Eagle's medium (Gibco-BRL, Grand Island, NY, USA) with $10 \%$ fetal bovine serum (Gibco-BRL), $100 \mathrm{U} / \mathrm{ml}$ penicillin and $100 \mathrm{mg} / \mathrm{ml}$ streptomycin at $37^{\circ} \mathrm{C}$ in an incubator with $5 \% \mathrm{CO}_{2}$ and $95 \%$ relative humidity. MCF-7 cells were trypsinized and passaged into 6-well or 96-well plates and were transfected with small interfering (si)RNAs to HGF when the cell density reached 30-50\% confluence. Lipofectamine 2000 (Invitrogen Life Technologies, Carlsbad, CA, USA) was used for transfection in accordance with the manufacturer's instructions.

Immunohistochemistry. Immunohistochemistry was performed using the two-step EnVision procedure (Dako, Copenhagen, Denmark). Briefly, each tissue section was deparaffinized, hydrated and then incubated with fresh $3 \%$ hydrogen peroxide $\left(\mathrm{H}_{2} \mathrm{O}_{2}\right)$ in methanol for $15 \mathrm{~min}$. Following rinsing with phosphate-buffered saline (PBS), antigen retrieval was performed by microwave treatment in $0.01 \mathrm{M}$ sodium citrate buffer $(\mathrm{pH} 6.0)$ at $100^{\circ} \mathrm{C}$ for $15 \mathrm{~min}$. Following this, tissue sections were incubated with primary monoclonal mouse-anti-human HGF antibodies (Santa Cruz Biotechnology, Inc., Santa Cruz, CA, USA) diluted in PBS containing $0.2 \%$ Triton $\mathrm{X}-100$ for $30 \mathrm{~min}$ at room temperature. Following rinsing with PBS, slides were incubated with the

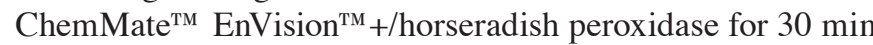
at room temperature. The reaction was visualized using ChemMate $^{\text {TM }}$ 3,3'-diaminobenzidine (DAB). Negative controls were prepared by substituting primary antibodies with PBS.

Immunohistochemical staining. Cells that generated a brown-colored polymeric oxidation product in their cytoplasm were defined as HGF-positive cells. Analysis of 10 discrete foci was performed in every section. The positive cells were graded in a blinded manner according to the following criteria: 0 , positive cells $\leq 5 \%$ and 1 , positive cells $>5 \%$. The stain intensity was graded according to the following criteria: 0 , no apparent brown-colored polymeric oxidation product and 1, clear brown-colored polymeric oxidation product. The final histoscore grade was calculated as the aggregate of positive cell grade plus the stain intensity grade: Negative, $0-1$ or positive, 2. As the estrogen receptor (ER) and progesterone receptor (PR) are expressed deep inside the nucleus, the DAB staining for them must be located within the nucleus. All experiments were repeated three times. Histological interpretation was performed independently by two pathologists blinded to the study conditions using an Olympus CX41 microscope (magnification, $\mathrm{x} 40$; Olympus Corporation, Tokyo, Japan).

Reverse-transcription quantitative polymerase chain reaction (RT-qPCR). Total RNA was extracted from the MCF-7 cells $48 \mathrm{~h}$ after transfection with siRNA-HGF using an RNeasy mini kit (Qiagen, Valencia, CA, USA). RT-qPCR was performed and the primer sequences used were as follows: HGF, forward 5'-CCACACGAACACAGCTATCGGGG-3' and reverse 5'-TGGGAGCAGTAGCCAACTCGGA-3'; GAPDH, forward 5'-GTCAGTGGTGGACCTGACCT-3' and reverse 5'-ACCTGGTGCRCAGTGRAGCC-3'. RNA without reverse transcriptase was also amplified and used as a negative control to rule out possible genomic DNA contamination. The PCR products were electrophoresed using a $1.2 \%$ agarose gel. The density of visualized bands was measured with a Tocan 240 imaging analysis device (Tocan, Shanghai, China).

Western blot analysis. The MCF-7 cells transfected with siRNA-HGF were lysed in lysis buffer. Following centrifugation at $15,000 \mathrm{x} \mathrm{g}$ for $15 \mathrm{~min}$, the protein concentration was measured using a BCA protein detection kit (Pierce Biotechnology, Inc., Rockford, IL, USA) and adjusted for equal loading. Subsequently, cell lysates were subjected to SDS-PAGE. Immunoreactivity was visualized by enhanced chemiluminescence. Quantification of immunoreactive bands was performed using Image Gauge software (Fuji Photo Film Co., Ltd., Tokyo, Japan).

MTT. The MCF-7 cells were passaged in 96-well plates and transfected with siRNA-HGF in five duplicates. MTT $(20 \mu \mathrm{l}$ of $5 \mathrm{mg} / \mathrm{ml}$ ) was added into the media 24,48 or $72 \mathrm{~h}$ after transfection, respectively. Dimethyl sulfoxide $(150 \mu \mathrm{l})$ was added following incubation in a culture hood for another $4 \mathrm{~h}$ and then agitated on an orbital shaker for $10 \mathrm{~min}$. The optical density value was detected using a 650-60 spectrophotometer (Hitachi, Ltd., Tokyo, Japan) at $490 \mathrm{~nm}$. In each group, different concentrations of EPI $(0.5,1,2,4$ and $8 \mu \mathrm{g} / \mu \mathrm{l})$ was added $72 \mathrm{~h}$ after transfection and the absorbance reading was performed again following incubation for another $24 \mathrm{~h}$. The experiment was repeated three times.

Assessment of chemotherapy effectiveness. The assessment of chemotherapy effectiveness was based on the Response Evaluation Criteria In Solid Tumors Guidelines established 
by the National Institutes of Health (Bethesda, MA, USA) and confirmed at 4 weeks: Complete response (CR), complete disappearance of all target lesions; partial response (PR), at least a $30 \%$ decrease in the sum of the longest diameter of target lesions; stable disease (SD), neither sufficient shrinkage to qualify for partial response nor sufficient increase to qualify for progressive disease; progressive disease (PD), at least a $20 \%$ increase in the sum of the longest diameter of target lesions. In the present study, 'effective' was defined as 'CR + PR' and 'ineffective' as 'SD + PD'.

Statistical analysis. All data were analyzed using SPSS 13.0 statistical software (SPSS, Inc., Chicago, IL, USA). Student's paired t-test and $\chi^{2}$ test were performed to analyze statistical significance in continuous variables and categorical variables, respectively. Survival rate was analyzed by a log-rank test. $\mathrm{P}<0.05$ was considered to indicate a statistically significant difference.

\section{Results}

Expression of HGF in human breast cancer tissues. In order to analyze the association between the expression of HGF and the clinical parameters in the present study, the positive rate of HGF in breast cancer tissues was determined. Breast cancer tissues were diagnosed by two pathologists and 92 of the 125 patients were diagnosed as infiltrating ductal carcinoma (Fig. 1A). The positive rate of HGF in human breast cancer tissues was $52 \%$ and was associated with TNM clinical stage, histological grade, lymph node metastasis $(\mathrm{P}<0.05)$, however, HGF was not associated with patient age and location, size and hormone receptor status of tumor $(\mathrm{P}>0.05$; Table I; Fig. 1B-E).

Correlation between HGF level and patient sensitivity to chemotherapy. In order to demonstrate the correlation between HGF level and patient sensitivity to chemotherapy, the effectiveness of chemotherapy in HGF positive or negative patients was compared. As is shown in Table II, the effeciency of chemotherapy in HGF negative patients was 90\%, which was significantly higher $(\mathrm{P}<0.05)$ than that in $\mathrm{HGF}$ positive patients $(68.75 \%)$.

mRNA expression of $H G F$ following transfection in MCF-7 cells. In order to confirm the inhibition of HGF mRNA expression by transfection with HGF-siRNA, RT-qPCR was performed in MCF-7 cells following transfection. As shown in Fig. 2, the mRNA expression of HGF was expressed in non-transfected MCF-7 cells. A similar expression level was found in the siRNA-GFP group. This was significantly downregulated following siRNA-HGF transfection (55\%), demonstrating that the mRNA expression of HGF was successfully inhibited.

Protein expression of HGF following siRNA transfection. To further confirm the inhibition of HGF at the protein level, western blot analysis was performed on the three groups. As shown in Fig. 3, HGF protein was expressed in non-transfected MCF-7 cells and a similar expression level was found in the siRNA-GFP group. This was significantly downregulated
Table I. Expression of HGF and its association with patient characteristics.

\begin{tabular}{|c|c|c|c|c|}
\hline \multirow{2}{*}{$\begin{array}{l}\text { Pathological } \\
\text { feature }\end{array}$} & \multicolumn{2}{|c|}{ HGF } & \multirow[b]{2}{*}{$\mathrm{X}^{2} / \mathrm{T}$} & \multirow[b]{2}{*}{ P-value } \\
\hline & - & + & & \\
\hline Age & 28 & 35 & 0.0529 & $>0.05$ \\
\hline$<44$ & 32 & 30 & & \\
\hline \multicolumn{5}{|l|}{$\geq 44$} \\
\hline \multicolumn{5}{|l|}{ Location } \\
\hline Left & 27 & 36 & 1.3459 & $>0.05$ \\
\hline Right & 33 & 29 & & \\
\hline \multicolumn{5}{|c|}{ Tumor size (cm) } \\
\hline$<5$ & 37 & 50 & 3.4322 & $>0.05$ \\
\hline$\geq 5$ & 23 & 15 & & \\
\hline \multicolumn{5}{|c|}{ Histological grade } \\
\hline I-II & 50 & 44 & 5.4597 & $<0.05$ \\
\hline III & 10 & 21 & & \\
\hline \multicolumn{5}{|c|}{ TNM clinical stage } \\
\hline I-II & 51 & 36 & 12.9332 & $<0.01$ \\
\hline III & 9 & 29 & & \\
\hline \multicolumn{5}{|c|}{ Lymph node metastasis } \\
\hline+ & 14 & 38 & 15.8475 & $<0.01$ \\
\hline- & 46 & 27 & & \\
\hline \multicolumn{5}{|l|}{ ER } \\
\hline+ & 33 & 43 & 1.6286 & $>0.05$ \\
\hline- & 27 & 22 & & \\
\hline \multicolumn{5}{|l|}{ PR } \\
\hline+ & 35 & 36 & 0.1106 & $>0.05$ \\
\hline- & 25 & 29 & & \\
\hline
\end{tabular}

HGF, hepatocyte growth factor; ER, estrogen receptor; PR, progesterone receptor.

Table II. Correlation between HGF and chemotherapy sensitivity.

\begin{tabular}{lcccccc}
\hline Items & CR & PR & SD & PD & Efficiency (\%) & P-value \\
\hline HGF+ & 3 & 19 & 8 & 2 & 68.75 & $<0.05$ \\
HGF- & 7 & 20 & 2 & 1 & 90 & $<0.05$ \\
\hline
\end{tabular}

HGF, hepatocyte growth factor; CR, complete response; PR, partial response; $\mathrm{SD}$, stable disease; $\mathrm{PD}$, progressive disease.

following siRNA-HGF transfection, indicating that the protein expression of HGF was successfully inhibited.

Proliferation assay following HGF-siRNA transfection and EPI co-culture. In order to determine whether HGF can affect cell proliferation, an MTT assay was performed. As shown in Fig. 4, the cell inhibition rate increased following the downregulation of HGF by HGF-siRNA transfection. The inhibitory 


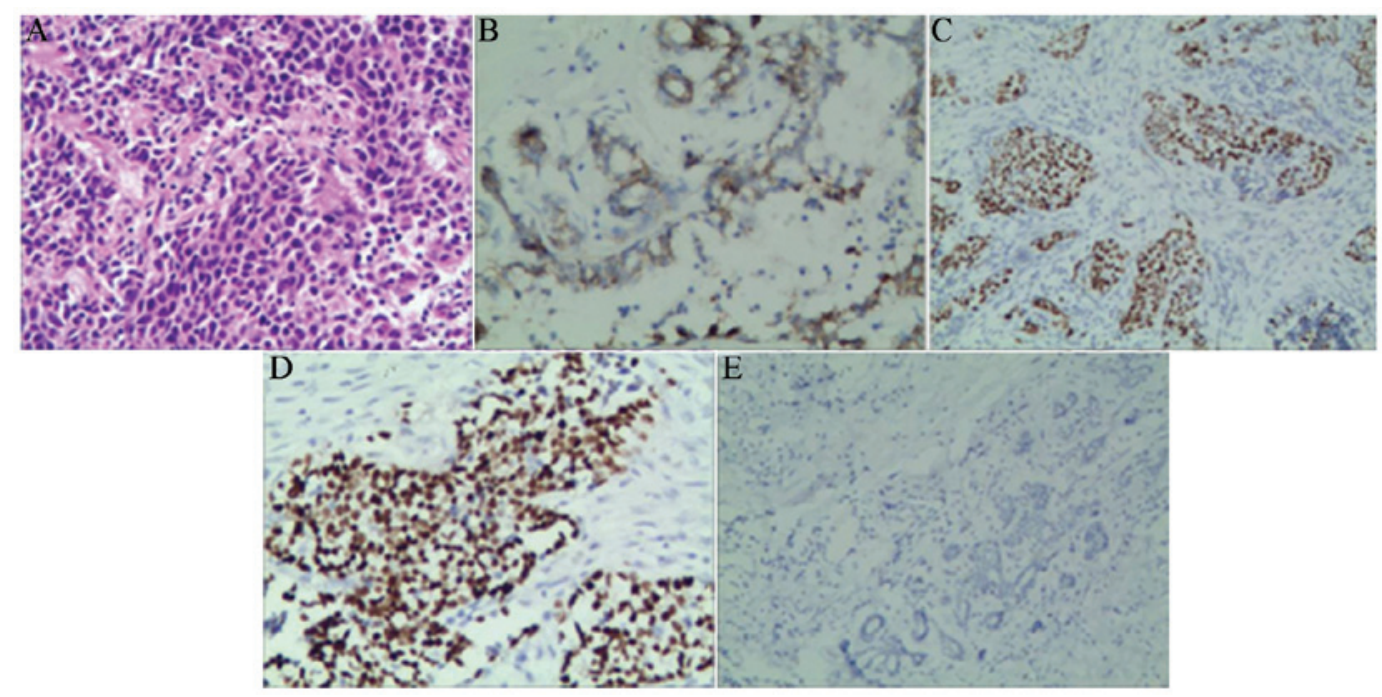

Figure 1. Immunohistochemistry analysis of breast cancer tissues (magnification, x400). (A) Hematoxylin and eosin staining of infiltrating ductal carcinoma. (B-D) Typical expression of hepatocyte growth factor, the estrogen receptor and progesterone receptor in breast cancer tissues. (E) Negative expression of HGF in the adjacent breast tissue.

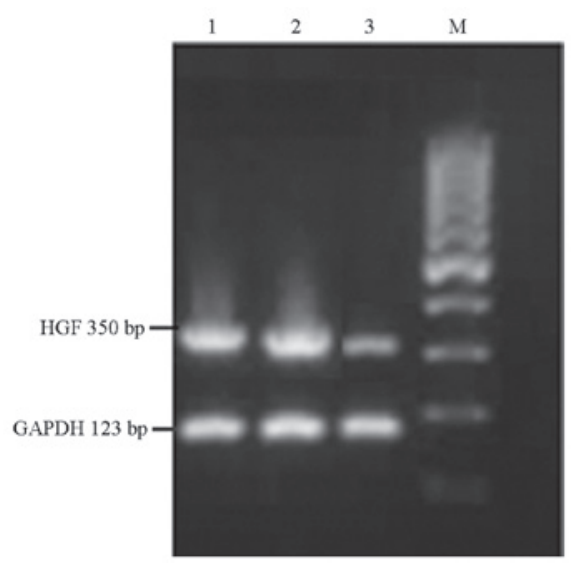

Figure 2. Reverse transcription-quantitative polymerase chain reaction analysis of HGF mRNA expression in MCF-7 cells. Lane 1, non-transfected MCF-7 cells; lane 2, siRNA-GFP-transfected MCF-7 cells; lane 3 , siRNA-HGF-transfected MCF-7 cells; M, DNA marker. mRNA expression of HGF was significantly downregulated following siRNA-HGF transfection.

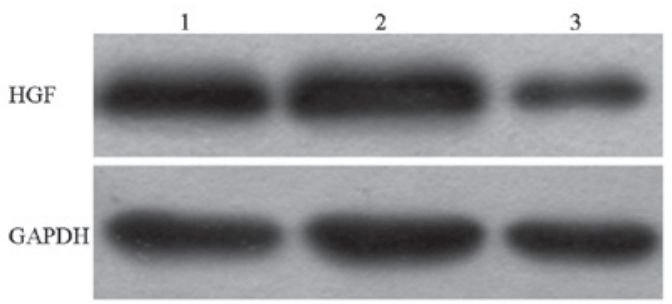

Figure 3. Western blot analysis of HGF expression in MCF-7 cells. Lane 1, non-transfected MCF-7 cells; lane 2, siRNA-GFP-transfected MCF-7 cells lane 3, siRNA-HGF-transfected MCF-7 cells. The results demonstrated that the protein level of HGF was significantly downregulated following siRNA-HGF transfection. HGF, hepatocyte growth factor; siRNA, small interfering RNA.

rate after 24,48 and $72 \mathrm{~h}$ was $54.33,78.09$ and $65.33 \%$, respectively, and was significantly higher than that of non-transfected and siRNA-GFP transfected groups ( $\mathrm{P}<0.05$; Fig. 4).

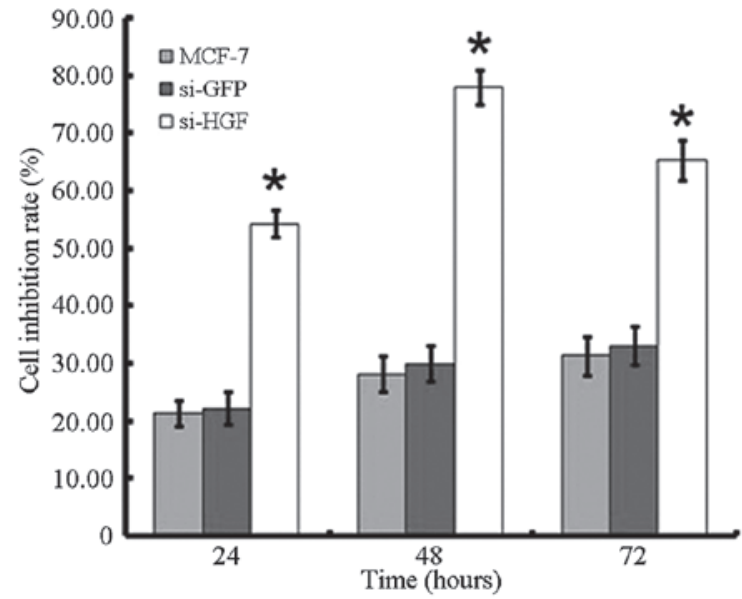

Figure 4. MTT assay of cell proliferation. Cell proliferation was markedly inhibited following siRNA transfection. The inhibitory rate after 24, 48 and $72 \mathrm{~h}$ was $54.33,78.09$ and $65.33 \%$, respectively, and was significantly higher than that of the non-transfected and siRNA-GFP-transfected groups $(\mathrm{P}<0.05)$. HGF, hepatocyte growth factor; siRNA, small interfering RNA.

As sensitivity to EPI in MCF-7 cells can represent cell sensitivity to chemotherapy, the sensitivity to EPI in the three groups was compared. The cell viability in the siRNA-HGF transfected group was significantly lower than the non-transfected and siRNA-GFP transfected groups, indicating that the expression of HGF has a vital role in cell survival when co-cultured with EPI (Fig. 5).

Correlation between HGF level and prognosis of breast cancer patients. Correlation analysis was performed to characterize the association between the expression of HGF and patient survival time. As shown in Fig. 6, the 5-year survival rate of HGF positive patients was $78 \%$, which was significantly lower $(\mathrm{P}<0.05)$ than that of HGF negative patients $(90 \%)$. The correlation analysis suggested that HGF may be an important factor in patient survival in breast cancer. 


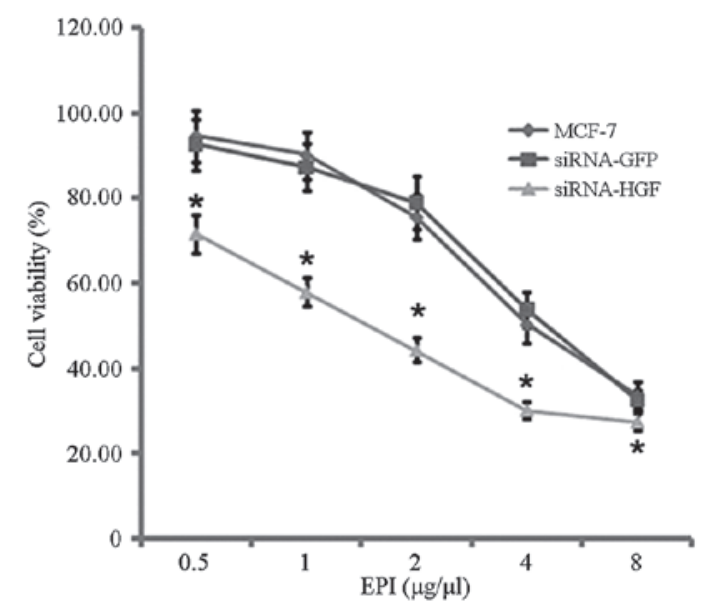

Figure 5. MTT assay of cell chemosensitivity to EPI. Following co-culture with EPI, the cell viability in the siRNA-HGF transfected group was significantly lower than the control groups. HGF, hepatocyte growth factor; siRNA, small interfering RNA; EPI, epirubicin.

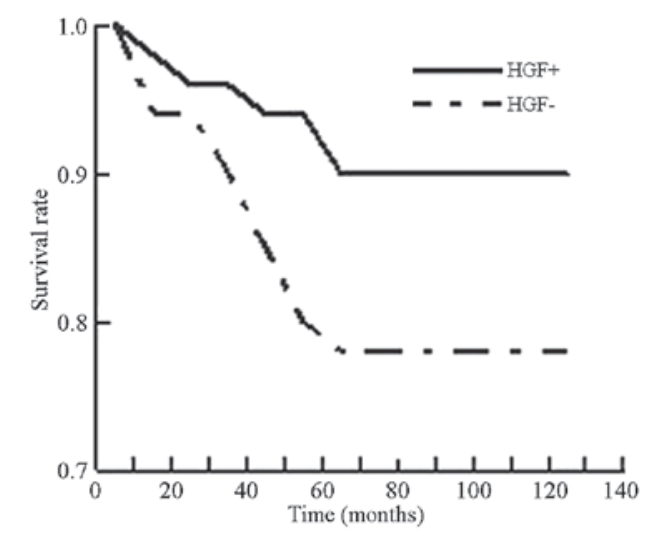

Figure 6. Correlation analysis of the association between expression of HGF and 5-year survival rate. The 5-year survival rate of HGF positive patients was $78 \%$, which was significantly lower $(\mathrm{P}<0.05)$ than that of HGF negative patients (90\%). HPG, hepatocyte growth factor.

\section{Discussion}

Breast cancer is clinically characterized as having a high morbidity, low chance of success through surgery alone, high recurrence and metastasis rate, poor prognosis and resistance to chemotherapy. It remains the most frequently diagnosed cancer and the leading cause of cancer-associated mortality among females. Thus, it is important to investigate the factors that can be used to predict metastasis, prognosis and sensitivity to chemotherapy post-surgically. HGF is a type of multifunctional peptide factor that is secreted by epithelial cells. It promotes the process of proliferation, migration, invasion and angiogenesis of various types of tumor $(3,4,12)$. It has been widely reported that high expression of HGF is closely associated with the prognosis of non-small cell lung cancer and colon cancer patients $(9,13)$. Yamashita et al (14) demonstrated that HGF was highly expressed in breast cancer and the expression level was closely associated with the prognosis of patients. Sheen-Chen et al (15) demonstrated that HGF is significantly correlated with the histological grade, clinical stage, tumor size and lymph node metastasis while investigating the association between the expression level of HGF and the pathological parameters in breast cancer patients. Parr et al (4) reported that breast cancer specimens express a significantly higher level of HGF, which indicates that the HGF regulatory system may be important in the progression of breast cancer. In the present study, the expression of HGF in breast cancer tissues of 125 patients was detected by immunohistochemistry. The results indicated that HGF was highly expressed in breast cancer tissues of patients and the positive rate was $52 \%$. The expression of HGF was not associated with patient age and location, size and hormone receptor status of tumor, however, HGF expression was associated with TNM clinical stage, histological grade, lymph node metastasis and prognosis. Furthermore, all 125 patients were followed up and their survival time and survival rate were compared and evaluated. The 5-year survival rate of HGF positive patients was significantly lower than that of HGF negative patients. This result indicated that HGF may be one of the essential predictors that contribute to the prognosis of breast cancer patients, which was consistent with a study by Eichbaum et al (16).

As it can improve the survival rate and surgical outcomes of breast cancer patients, neoadjuvant chemotherapy (NACT) has been used more and more widely in clinical practice. Nevertheless, few studies investigating the effect of NACT on HGF and HGF as a predictor for sensitivity to chemotherapy was insufficiently characterized. In order to avoid the unnecessary side effects of chemotherapy that patients may undergo and to improve the sensitivity to chemotherapy, defined valuable indicators are required to better understand NACT. Of the breast cancer patients in the present study, 62 were in the presurgical NACT group. Among them, the effective rate of chemotherapy in HGF negative patients was $~ 90 \%$ and was significantly higher than that in HGF positive patients $(68.75 \%)$, which suggested that HGF is closely associated with sensitivity to chemotherapy. Analogous results were reported by Lengyel et al (17). They identified that the HGF receptor, c-Met was closely associated with sensitivity to chemotherapy and of all the patients in the study, five converted to complete remission and the positive rate of c-Met was $20 \%$.

Furthermore, in vitro studies were performed to confirm that HGF was able to increase the sensitivity to chemotherapy in breast cancer patients. The HGF expression profile of MCF-7 cells was identified following the specific silence of HGF by siRNA. As shown in the RT-qPCR and western blotting results, HGF was significantly downregulated following siRNA transfection in MCF-7 cells, whose proliferation rate significantly decreased compared with the control groups. This confirmed that HGF had the capacity to enhance cell proliferation. By contrast, when co-cultured with EPI for $24 \mathrm{~h}$, the survival rate of transfected cells was significantly lower than that of the non-transfected group. In this manner, HGF has been verified to be important in resistance to EPI at the cellular level. The conclusion that HGF is associated with sensitivity to chemotherapy in breast cancer patients was also supported by other studies $(18,19)$. It was hypothesized that this function was associated with the activation of intracellular AKT, which is linked to cellular resistance to chemotherapeutic drugs (20). Future studies may determine the concrete mechanisms by which HGF functions during this process. 
In conclusion, HGF may be a promising, new therapeutic target for breast cancer and may enable clinical practitioners to better predict patient sensitivity to NACT and prognosis through detecting patient HGF levels. Although the present study demonstrated the possibility and availability of HGF as a useful predictor, understanding the mechanisms underlying the effect requires further investigation.

\section{Acknowledgements}

This study was supported by a grant from the Project of National Natural Science Funds and Youth Funds, China (grant no. 81000914).

\section{References}

1. Jemal A, Bray F, Center MM, Ferlay J, Ward E and Forman D: Global cancer statistics. CA Cancer J Clin 61: 69-90, 2011.

2. Wang L, Wang C, Su B, et al: Recombinant human PDCD5 protein enhances chemosensitivity of breast cancer in vitro and in vivo. Biochem Cell Biol 91: 526-531, 2013.

3. Singh-Kaw P, Zarnegar R and Siegfried JM: Stimulatory effects of hepatocyte growth factor on normal and neoplastic human bronchial epithelial cells. Am J Physiol 268: L1012-L1020, 1995.

4. Parr C, Watkins G, Mansel RE and Jiang WC: The hepatocyte growth factor regulatory factors in human breast cancer. Clin Cancer Res 10: 202-211, 2004.

5. Jiang W, Hiscox S, Matsumoto K and Nakamura T: Hepatocyte growth factor/scatter factor, its molecular, cellular and clinical implications in cancer. Crit Rev Oncol Hematol 29: 209-248, 1999.

6. El-Attar HA and Sheta MI: Hepatocyte growth factor profile with breast cancer. Indian J Pathol Microbiol 54: 509-513, 2011.

7. Gallego MI, Bierie B and Hennighausen L: Targeted expression of $\mathrm{HGF} / \mathrm{SF}$ in mouse mammary epithelium leads to metastatic adenosquamous carcinomas through the activation of multiple signal transduction pathways. Oncogene 22: 8498-8508, 2003.

8. Huang S, Ouyang N, Lin L, et al: HGF-induced PKC $\zeta$ activation increases functional CXCR4 expression in human breast cancer cells. PLoS One 7: e29124, 2012.
9. Siegfried JM, Weissfeld LA, Luketich JD, Weyant RL, Gubish CT and Landreneau RJ: The clinical significance of hepatocyte growth factor for non-small cell lung cancer. Ann Thorac Surg 66: 1915-1918, 1998.

10. Locatelli A, Lofgren KA, Daniel AR, Castro NE and Lange CA: Mechanisms of HGF/Met signaling to Brk and Sam68 in breast cancer progression. Horm Cancer 3: 14-25, 2012.

11. Woodbury RL, Varnum SM and Zangar RC: Elevated HGF levels in sera from breast cancer patients detected using a protein microarray ELISA. J Proteome Res 1: 233-237, 2002.

12. Hirose Y, Kojima M, Sagoh M, Murakami H, Yoshida K, Shimazaki K and Kawase T: Immunohistochemical examination of c-Met protein expression in astrocytic tumors. Acta Neuropathol 95: 345-351, 1998.

13. Takeuchi H, Bilchik A, Saha S, et al: c-MET expression level in primary colon cancer: a predictor of tumor invasion and lymph node metastases. Clin Cancer Res 9: 1480-1488, 2003.

14. Yamashita J, Ogawa M, Yamashita S, Nomura K, Kuramoto M, Saishoji T and Shin S: Immunoreactive hepatocyte growth factor is a strong and independent predictor of recurrence and survival in human breast cancer. Cancer Res 54: 1630-1633, 1994.

15. Sheen-Chen SM, Liu YW, Eng HL and Chou FF: Serum levels of hepatocyte growth factor in patients with breast cancer. Cancer Epidemiol Biomarkers Prev 14: 715-717, 2005.

16. Eichbaum MH, de Rossi TM, Kaul S, Bruckner T, Schneeweiss A and Sohn C: Serum levels of hepatocyte growth factor/scatter factor in patients with liver metastases from breast cancer. Tumour Biol 28: 36-44, 2007.

17. Lengyel E, Prechtel D, Resau JH, et al: C-Met overexpression in node-positive breast cancer identifies patients with poor clinical outcome independent of Her2/neu. Int J Cancer 113: 678-682, 2005.

18. Minuti G, Cappuzzo F, Duchnowska R, et al: Increased MET and HGF gene copy numbers are associated with trastuzumab failure in HER2-positive metastatic breast cancer. Br J Cancer 107: 793-799, 2012.

19. Parr C and Jiang WG: Hepatocyte growth factor activation inhibitors (HAI-1 and HAI-2) regulate HGF-induced invasion of human breast cancer cells. Int J Cancer 119: 1176-1183, 2006.

20. Knuefermann C, Lu Y, Liu B, et al: HER2/PI-3K/Akt activation leads to a multidrug resistance in human breast adenocarcinoma cells. Oncogene 22: 3205-3212, 2003. 